\title{
Particle Beam Waist Location in Plasma Wakefield Acceleration: Methods, Data and Discussion
}

\author{
Adrian Down \\ Department of Physics \\ University of California \\ Berkeley, California 94720-7300 USA
}

Received: September 27, 2006 Accepted: January 2, 2007

\begin{abstract}
The role of beam waist location in interactions between a plasma and a particle beam is not yet fully understood. Nonlinear effects with the plasma make an analysis of such interactions difficult. Five simulations are presented in this report, with the waist location of a beam of ultra-relativistic electrons propagating through one meter of self-ionized lithium plasma. The simulation parameters are chosen to model the recent experiment 167 at the Stanford Linear Accelerator, relevant to the design of future plasma wakefield accelerating afterburners. It is found that beams focused near the point of entry into the plasma propagate further into the plasma and accelerate witness particles to a greater maximum energy before disintegrating. These results could indicate that ion channel formation is dependent on the drive beam waist location and that the plasma accelerating medium can have an observable effect on the focusing of the drive beam.
\end{abstract}

Editorial Note: The research outlined below is the second part of a report that began in the March 2007 issue.

\section{METHODS: E-167 EXPERIMENT}

The E-167 experiments are a series of tests of technology to be used in future afterburners. These tests began in 2005 at the Stanford Linear Accelerator Center (SLAC), and are currently in operation. Complete data from the experiments is forthcoming; however, preliminary results have already demonstrated energy doubling of some particles in the $42 \mathrm{GeV}$ drive beam over a distance of 1 meter.

\section{a. Apparatus}

The E-167 experiments are being carried out at the Final Focus Test Beam (FFTB) facility at SLAC. This recently completed facility has the potential to create electron bunches with a minimum length of about $12 \mu \mathrm{m}$. These unprecedentedly short electron bunches can lead to higher radial electric fields in PWFA experiments, as predicted by equation (8) [see Down, part 1]. More precise control over the bunch length also allows the current profile of the drive and witness beams to be shaped so as to maximize the acceleration gradient experienced by the witness bunch [4].

Full details of the E-167 experimental apparatus are presented in [4]. A schematic diagram of the experimental apparatus is shown in Figure 8. Here we will summarize some of the relevant experimental aspects.

The $42 \mathrm{GeV}$ SLAC electron bean is passed though a 1 meter tube containing lithium vapor. Lithium is chosen because the large ionization potential of the second bound electron of the lithium atom prevents significant secondary ionization from occurring when the drive beam is passed through the lithium vapor. Multiple ionization can lead to uncertainty in the electron density in the plasma and undesirable trapping of plasma electrons [15], as described earlier.

The lithium is initially electrically neutral and is vaporized by heating pellets of solid lithium. The lithium vapor becomes self-ionized upon impact with the SLAC electron beam. This drive beam simultaneously ionizes the lithium vapor and excites a wakefield structure.

The lithium vapor is bounded on each end by a helium buffer gas, and the 


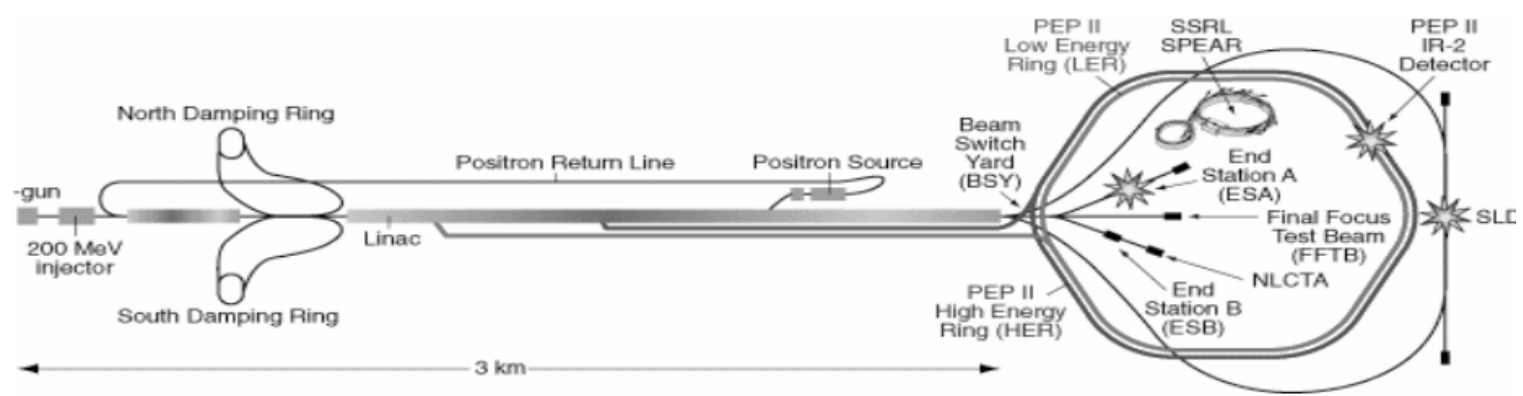

Figure 8. Schematic diagram of the Stanford Linear Accelerator (SLAC). The E-167 experiments are carried out in the Final Focus Test Beam (FFTB) facility.

density of the plasma created from the lithium vapor can be controlled by adjusting the vapor density in the tube, a process which is precise and highly stable [4]. The stability of the vapor system implies that effects observed when the drive beam is passed through the plasma are likely due to properties of the drive beam itself and not due to the initial condition of the lithium plasma [15].

\section{b. Diagnostics}

The bunches used in the E-167 experiments are shorter than those used in previous experiments. New data collection techniques are being developed that operate at the small time scales necessitated by short drive bunches.

The energy of the beam before entering the plasma is inferred from radiation emitted when the drive beam is deflected using chicane magnets. The synchrotron radiation resulting upon deflection is imaged on florescent Ce:YAG crystals. The amount of deflection experienced by the is beam particles, and hence the amount of emitted radiation, is proportional to the energy of the particles. Thus the observed radiation spectrum provides an unambiguous measurement of the energy profile of the beam [4].

Measurement of the bunch length is particularly difficult due to the short bunch lengths used in the E-167 experiment. Traditional measurement techniques cannot be used with high precision. Instead, beam particles are passed through a conducting foil, and the resulting coherent transition radiation is used as a measure of the longitudinal profile of the beam. A complete description can be found in [16].

After emerging from the plasma, the beam is passed through an imaging spectrometer and resulting spectrum is passed through a Cherenkov radiator. The emitted Cherenkov radiation is imaged on a piece of silica aerogel. As with the measurement of the initial beam energy, the radiation spectrum is interpreted as a measurement of the beam energy profile.

The energy profile observed after the beam has passed through the plasma can be calibrated against the initial beam energy by running the experimental apparatus with no lithium vapor. For calibration, the lithium vapor in the beam line can be exchanged for a vacuum in a matter of seconds [4].

c. Results

The E-167 experiments demonstrate the possibility of achieving unprecedented acceleration gradients. A small number of particles of the $42 \mathrm{GeV}$ SLAC drive beam can double in energy to over $80 \mathrm{GeV}$ in a distance of $1 \mathrm{~m}$.

The energy spectra observed after the drive beam passes through the lithium plasma demonstrates unexpected behavior at low energy, however. As shown in Figure 9 , there is a small tail of particles extending to very low energies. This monotonic tail of low energy particles is not reproduced by simulations, as shown in Figure 21.

An explanation of the observed low energy spectrum could provide insight into the physical processes operative in PWFA. Studying these low energy particles could also help to improved simulations if there 


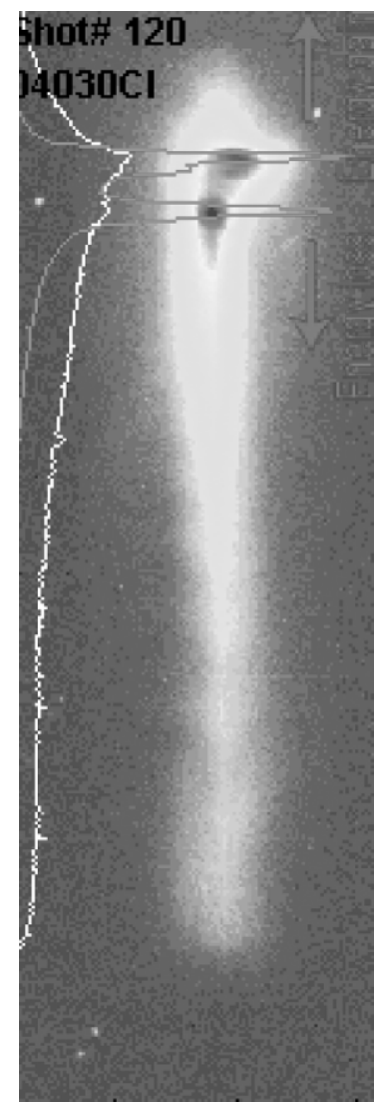

Figure 9. Beam energy spectrum observed in E-167 experiments, as inferred from Cherenkov radiation upon deflection of beam. Energy is proportional to vertical position, with higher energies at greater vertical position. Vertical scale is not linear. The highest energy particles doubled in energy to over $80 \mathrm{GeV}$. The lowest energy particles form a monotonic tails that was not reproduced in our simulations.

are effects that are not currently modeled correctly.

Simulations presented in this paper are not able to reproduce the observed spectrum of low energy particles by varying beam waist position. Results of this study indicate that the tail of low energy particles show in Figure 9 is likely due to trapping of plasma electrons.

\section{METHODS: QUICKPIC SIMULATION ALGORITHM}

a. Introduction

Effective models of plasma based accelerators must simulate the evolution of the drive beam, the generation and evolution of the wake, and the acceleration of the witness bunch. Due to nonlinear effects, the tasks require particle based models. The particle-in-cell (PIC) algorithm is one such model that has shown good agreement with experimental results [8]. However, the full PIC model is too computationally intensive for large simulations. The simulations presented in this paper would take over $10^{5}$ computer hours each if run using the full PIC model.

The QuickPIC algorithm reduces the necessary computational time by taking advantage of the different time scales of the evolution of the driver and the trailing beam. The drive beam evolves much slower than the wakefield structure, and so the QuickPIC algorithm operates on a quasi-static model in which the drive beam is held constant over a specified distance while the wakefield is evolved at intermediate steps.

\section{b. Quasi-static PIC Equations}

In PIC simulations, particles are located on a spatial grid. The charge and current densities calculated at grid points are used to advance the fields calculated from Maxwell's equations. The fields are used to advance the position and velocity of the particles using relativistic equations of motion.

The QuickPIC simulation algorithm begins with Maxwell's equations in the Lorentz gauge (in CGS units),

$$
\begin{aligned}
& \left(\frac{1}{c^{2}} \frac{\partial}{\partial t^{2}}-\nabla^{2}\right) \phi(x, y, z, t)=4 \pi \rho(x, y, z, t) \\
& \left(\frac{1}{c^{2}} \frac{\partial}{\partial t^{2}}-\nabla^{2}\right) \vec{A}(x, y, z, t)=\frac{4 \pi}{c} \vec{J}(x, y, z, t)
\end{aligned}
$$

where $\phi$ is related to the electric potential $V$, expressed in SI units, by $\phi=4 \pi \varepsilon_{0} V$.

The motion of plasma particles is determined from the Lorentz force equation,

$$
\frac{d \vec{P}}{d t}=q(\vec{E}+\vec{\beta} \times \vec{B})
$$




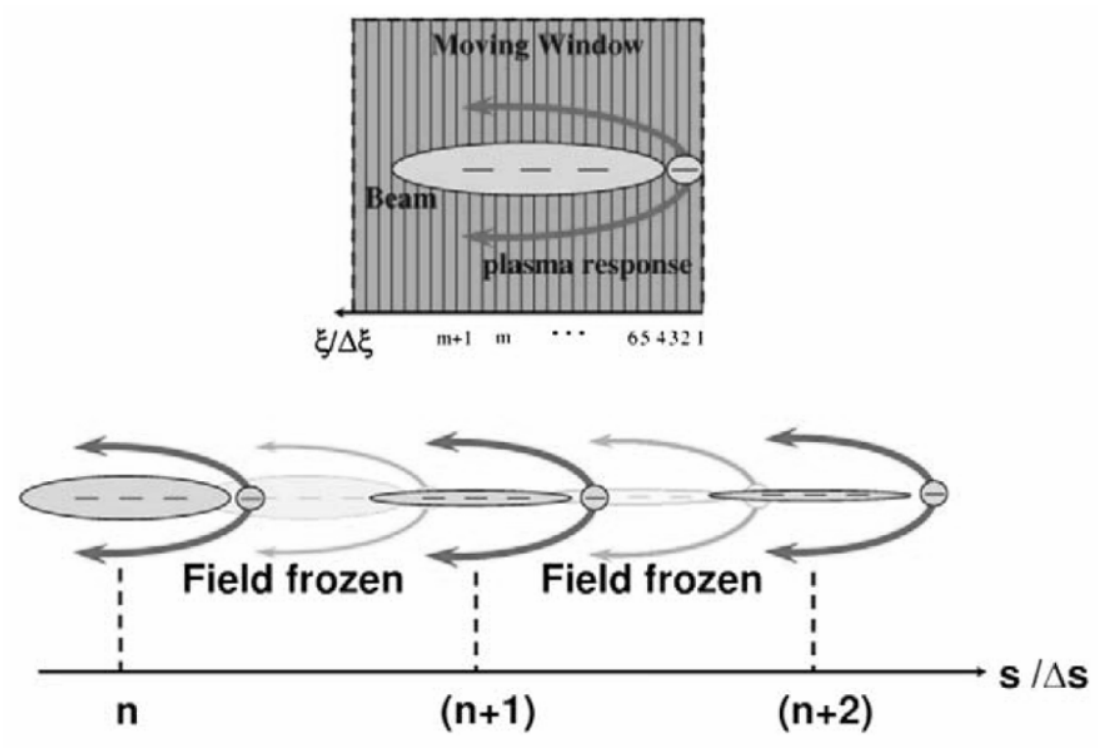

Figure 10. Cartoon of quasi-static timescale separation in the QuickPIC algorithm. The drive beam evolves more slowly than the wakefield structure. In the quasi-static approximation, the fields associated with the drive beam are held fixed as the beam is propagated over a distance $\Delta s$, whereas the wakefield structure is updated on a smaller timescale $\Delta \xi$.

where $\vec{\beta}=\vec{v} / \mathrm{c}$.

It is convenient to transform to coordinates that reflect the different scales of the evolution of the drive beam and the wakefield structure,

$$
S=Z \quad \xi=c t-Z
$$

with the accompanying Jacobians,

$$
\frac{\partial}{\partial z}=-\frac{\partial}{\partial \xi} \quad \frac{\partial}{c \partial t}=\frac{\partial}{\partial \xi}
$$

$\xi$ acts as a fast time scale associated with the drives and the development of wake; $s$ acts as a slow time scale associated with the evolution of shape of the driver.

In these transformed coordinates, the quasi-static approximation is,

$$
\frac{\partial}{\partial s}<<\frac{\partial}{\partial \xi}
$$

In this approximation, the wakefield is "frozen" as the drive beam is propagated over a number of small time steps $\Delta \xi$. The drive beam is updated on the slower time scale $\Delta s$. A cartoon schematic of how this approximation is used by the QuickPIC algorithm is shown in Figure 10.

\section{c. Beam Evolution}

The drive beam evolves on the time scale $\Delta s$ and so it will be convenient to write the equations governing the evolution of the drive beam in terms of the parameter $s$. Beam particles are treated as being ultrarelativistic, so that the velocity of the beam particles $v_{b}$ is approximately equal to $c \hat{z}$. Thus longitudinal position can alternatively be used as a measure of time,

$$
\frac{d s}{d t} \approx c \Rightarrow \frac{d}{d t} \approx c \frac{d}{d s}
$$

Equations (15) and (16) govern the evolution of the wakefield structure. Using (21), the longitudinal dependence in these equations cancels, leaving

$$
\begin{aligned}
\nabla_{\perp}^{2} \phi(x, y, s, \xi) & =-4 \pi \rho(x, y, s, \xi) \\
\nabla_{\perp}^{2} \vec{A}(x, y, s, \xi) & =-\frac{4 \pi}{c} \vec{J}(x, y, s, \xi)
\end{aligned}
$$




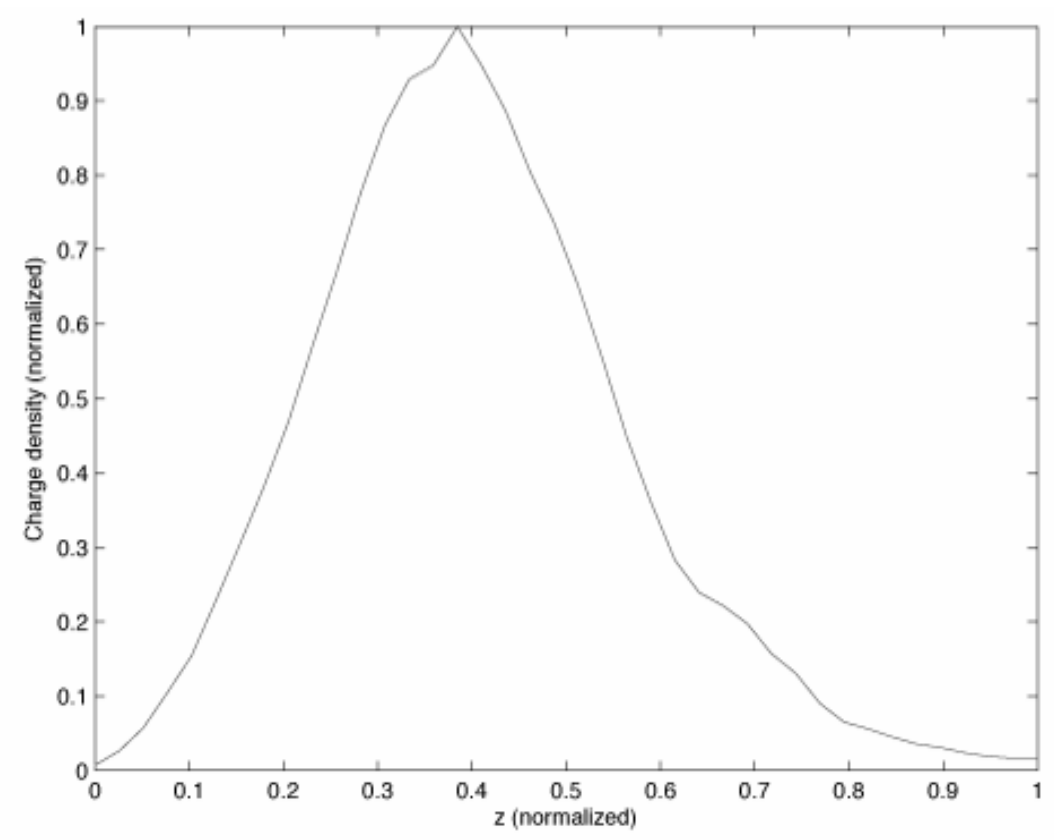

Figure 11. Drive beam longitudinal density profile, in normalized units.

The Lorentz gauge condition can be expressed in $s, \xi$ coordinates using (19),

$$
\vec{\nabla}_{\perp} \cdot \vec{A}_{\perp}(x, y, s, \xi)=-\frac{\partial}{\partial \xi} \psi(x, y, s, \xi)
$$

where $\psi=\phi-A_{z}$.

Equation (17) describes the evolution of the momenta of beam particles. Before evaluating this equation, it is useful to recast the fields in terms of the electromagnetic potentials,

$$
\vec{E}=-\vec{\nabla} \phi-\frac{\partial \vec{A}}{c \partial t} \quad \vec{B}=\vec{\nabla} \times \vec{A}
$$

Using (25) and (19), (17) becomes

$$
\frac{d \vec{P}_{b \perp}}{d s}=-\frac{q_{b}}{c} \vec{\nabla}_{\perp} \psi \quad \frac{d P_{b s}}{d s}=\frac{q_{b}}{c} \frac{\partial \psi}{\partial \xi}
$$

The evolution equations for the transverse positions of the beam particles can be obtained from the definition of the relativistic momentum,

$$
\vec{P}_{b \perp}=\gamma m c \vec{\beta}
$$

where $\beta$ is related to position,

$$
\vec{\beta}=\frac{\vec{v}}{c}=\frac{d \vec{x}}{c d t}=\frac{d \vec{x}}{d s} .
$$

Substituting (28) into (27) gives

$$
\frac{d \vec{x}_{b \perp}}{d s}=\frac{\vec{P}_{b \perp}}{\gamma m c} .
$$

d. Plasma Wakefield Evolution

The wakefield structure evolves on the shorter timescale corresponding to $\xi$. Hence we recast the Lorentz force equation (17) in terms of $\xi$,

$$
\frac{d \vec{P}_{p \perp}}{d s}=\frac{d \vec{P}_{p \perp}}{d t} \frac{d t}{d s} \frac{d s}{d \xi} .
$$

The necessary derivatives are,

$$
\begin{gathered}
\frac{d \xi}{d s}=\frac{d}{d s}(c t-z)=\frac{d}{c d t}(c d t)-\frac{d z}{d s} \\
\Rightarrow \frac{d \xi}{d s}=1-\frac{P_{z}}{\lambda m c}=1-\beta_{z}
\end{gathered}
$$

Substituting (31) into (30), 

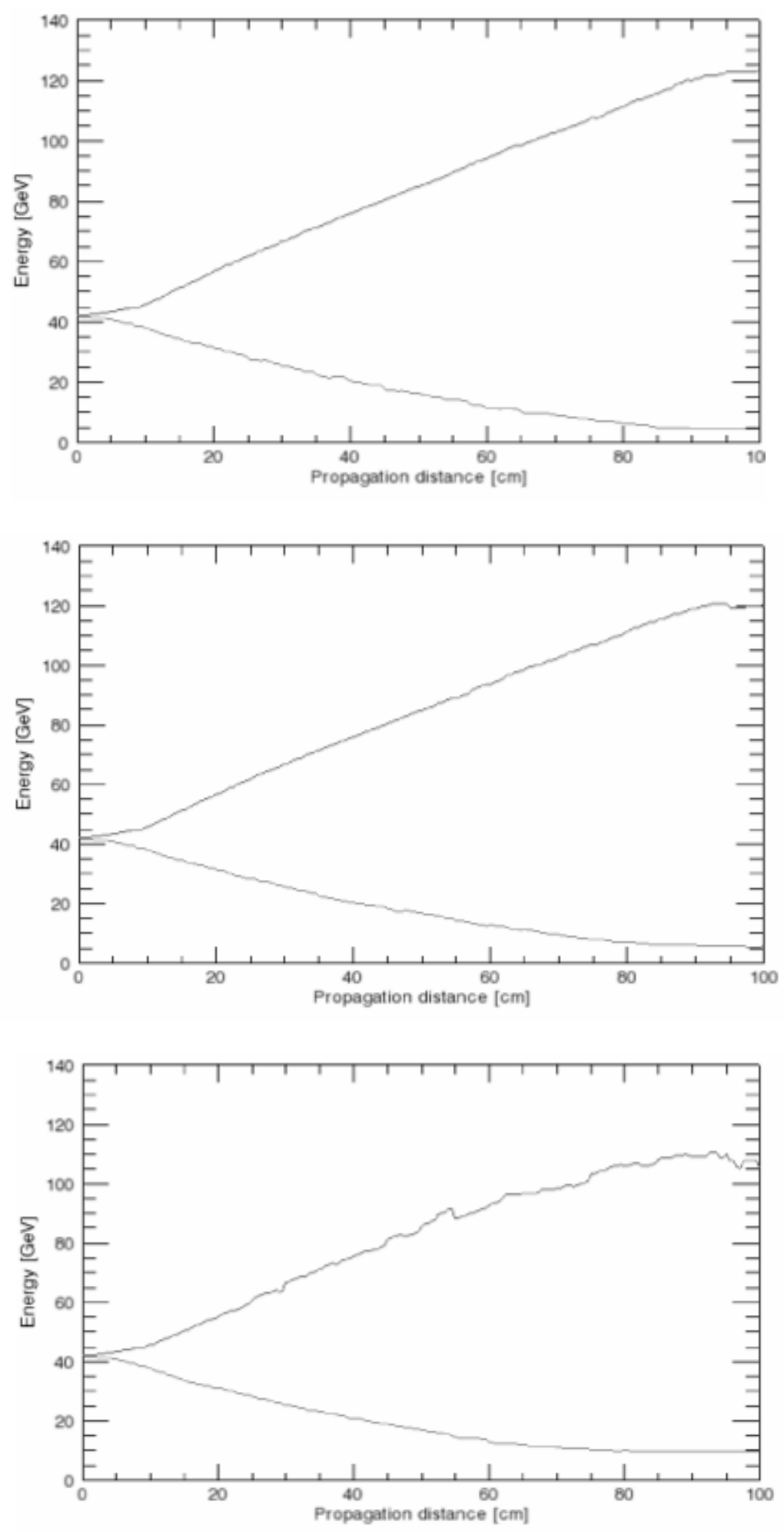

Figure 12 (a). Maximum and minimum energies of beam particles as a function of propagation distance. Top: waist position $=0 \mathrm{~cm}$; Middle: waist position $=5 \mathrm{~cm}$; Bottom: waist position $=15$ $\mathrm{cm}$. The upper curves in each figure show the maximum energy and the lower show the minimum energy. 

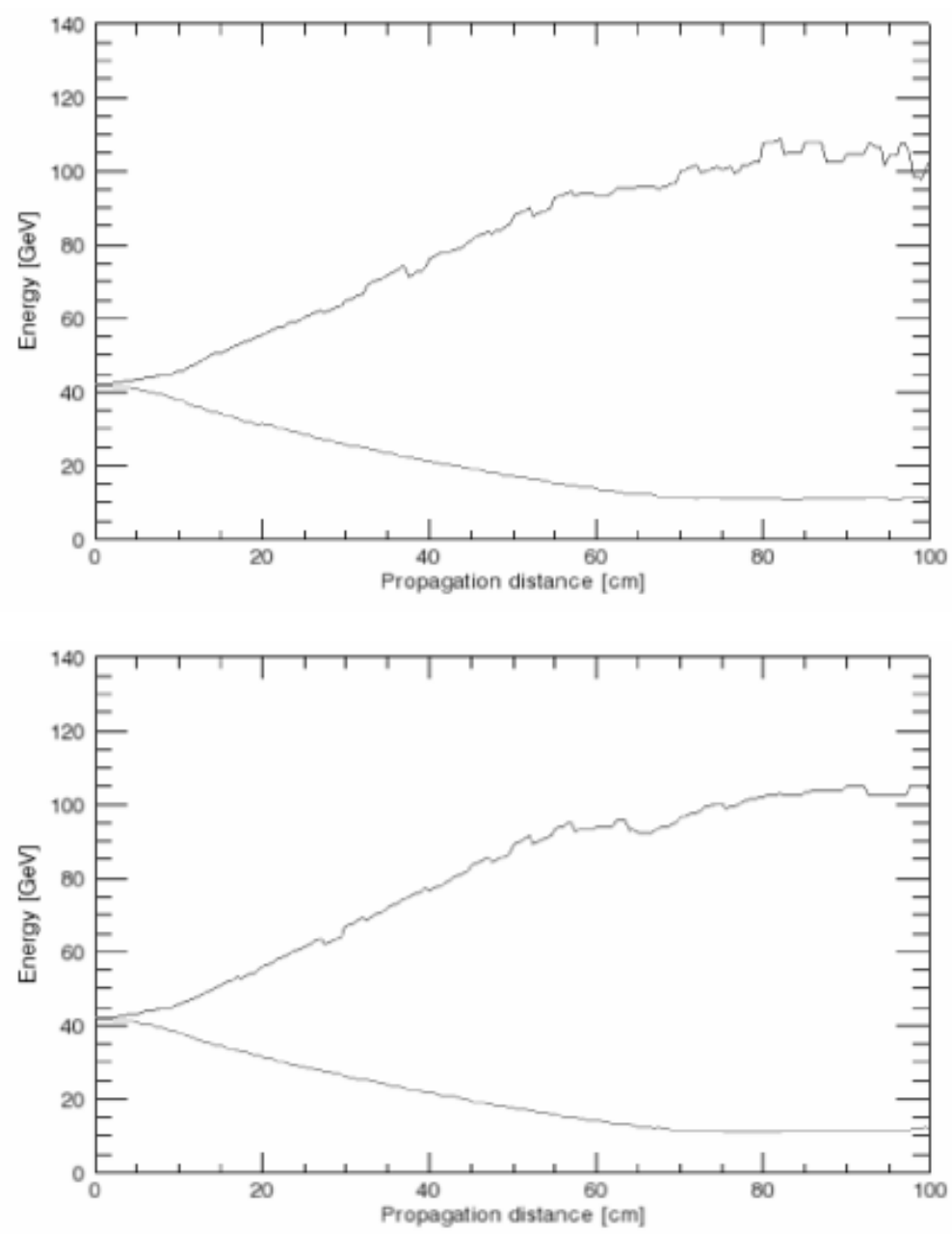

Figure 12 (b). Maximum and minimum energies of beam particles as a function of propagation distance. Top: waist position $=20 \mathrm{~cm}$; Bottom: waist position $=25 \mathrm{~cm}$. The upper curves in each figure show the maximum energy and the lower show the minimum energy. For (a) and (b): Beams focused near $z=0$ achieve greater energy gain than those focused at larger $z$. Energy maxima change at nearly a constant rate in shallowly focused beams, whereas maxima appear to asymptote for more deeply focused beams. No beams appear to be limited by pump depletion.

$$
\frac{d \vec{P}_{p \perp}}{d \xi}=\frac{q_{p}}{c-v_{p z}}\left[\vec{E}_{\perp}+\left(\vec{\beta}_{p} \times \vec{B}\right)_{\perp}\right]
$$

The longitudinal momentum of the plasma particles can be obtained from a constant of the motion [8],

$$
\gamma_{p}-\frac{P_{p \perp}}{m c}=1-\frac{q_{p} \psi}{m c^{2}}
$$

where $\gamma_{p}$ is the relativistic factor of the plasma particles.

The equation describing the evolution of the position of the plasma particles is obtained from (28) and (31),

$$
\frac{d \vec{x}_{p \perp}}{d \xi}=\frac{d \vec{x}_{p \perp}}{d s} \frac{d s}{d \xi}=\frac{\beta_{p \perp}}{1-\beta_{p z}} .
$$

Equations (32) and (34) determine the trajectory of each plasma particle as it is swept over by the drive beam.

\section{e. Deposition Scheme}

The deposition scheme refers to the method by which the charge and current densities, $\rho$ and $\vec{J}$, are determined from the charge, position, and momentum of 


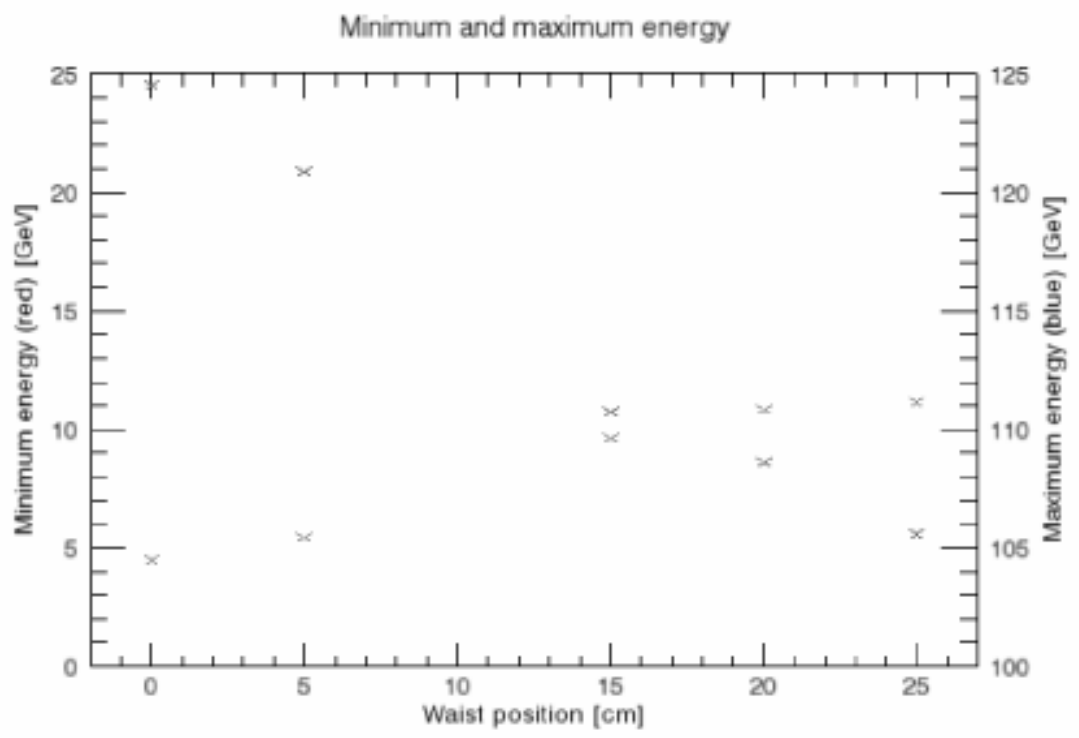

Figure 13. Energy extrema of greatest magnitude achieved over total propagation distance. Left and right vertical scales are in $\mathrm{GeV}$, with the left scale measuring the minimum energy and the right maximum energy. The minimum energy begins at left $(0 \mathrm{~cm})$ at just under $5 \mathrm{GeV}$; the maximum energy starts at left $(0 \mathrm{~cm})$ near $25 \mathrm{GeV}$. Energy maxima show a clear downward trend with increasing waist position. Energy minima display an upwards trend with increasing waist position.

individual particles. The method begins with the discreet version of conservation of charge,

$$
\begin{aligned}
0= & \underbrace{\frac{\partial}{\partial t} \sum_{i} Q_{i} \delta\left(\vec{x}-\vec{x}_{i}(t)\right)}_{\frac{\partial \rho}{\partial t}} \\
& +\underbrace{\vec{\nabla} \cdot\left[\sum_{i} Q_{i} \vec{v}_{i} \delta\left(\vec{x}-\vec{x}_{i}(t)\right)\right]}_{\vec{\nabla} \cdot \vec{J}} .
\end{aligned}
$$

The perpendicular and $\hat{z}$ components in the $\delta$ functions can be separated. Integrating (35) over $z$ within both summations, $z$ is set to $z_{i}(t)=v_{z i} t$.

It is desirable to recast the time derivative in the first term of (35) using the relevant time scale $\xi$. For a given particles, $\mathrm{z}$ is determined by the integration over the $\delta$ function.

$$
\xi=c t-z=c t-v_{z i} t
$$

$$
\begin{aligned}
& \Rightarrow \frac{\partial \xi}{\partial t}=c-v_{z i} \\
& \Rightarrow \frac{\partial}{\partial t}=\left(c-v_{z i}\right) \frac{\partial}{\partial \xi}
\end{aligned}
$$

For the second term in (35), the $\hat{s}$ component of the derivative is negligible in the quasi-static approximation. Hence (35) can be written,

$$
\begin{aligned}
0 & =\frac{\partial}{\partial \xi} \sum_{i} Q_{i}\left[1-\beta_{z i}(\xi)\right] \delta\left(\vec{x}_{\perp}-\vec{x}_{\perp i}(\xi)\right) \\
& +\vec{\nabla}_{\perp} \cdot\left[\sum_{i} Q_{i} \frac{v_{\perp i}(\xi)}{c} \delta\left(\vec{x}_{\perp}-\vec{x}_{\perp i}(\xi)\right)\right] .
\end{aligned}
$$

Equation (37) can be integrated over all $x$ and $y$. The first term depends only on the longitudinal coordinate, and so the integration removes the $\delta$ function. The argument of the divergence in the second term is equal to 0 at the bounds of 


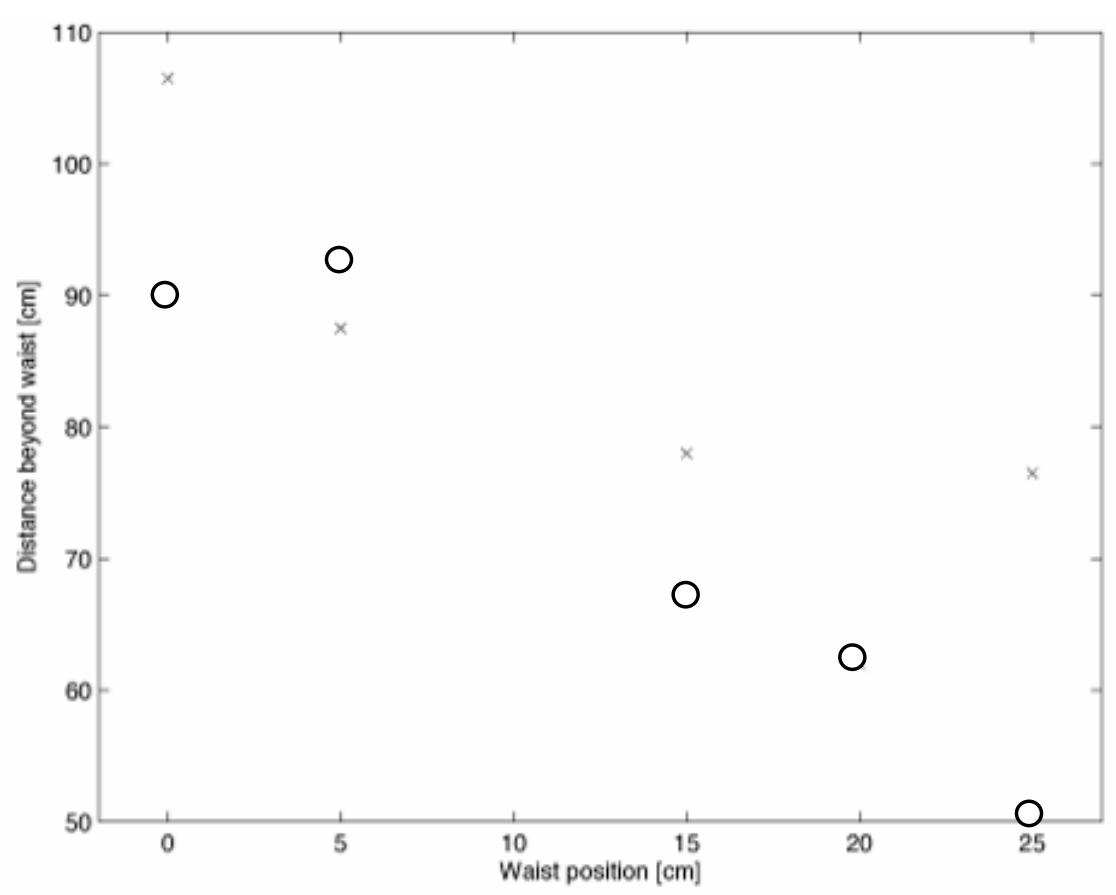

Figure 14. Distance beyond waist at which the maximum $(x)$ and minimum $(\mathbf{O})$ energies are achieved, for the energy extrema plotted in Figure 13. Distance of extrema after beam waist generally decreases as waist location is increased. The decrease in distance at which extrema are achieved could be indicative of limitations due to poor ion channel formation by deeply focused beams with initially large transverse spot size.

integration, and so the integral of this term is equal to 0 . After integration, (37) becomes

$$
0=\frac{\partial}{\partial \xi} \sum_{i} Q_{i}\left[1-\beta_{z i}(\xi)\right]
$$

Thus the quantity that is preserved as $\xi$ is advanced is the effective charge

$$
q_{i}=Q_{i}\left[1-\beta_{z i}(\xi)\right]
$$

and not the absolute charge $Q_{i}$. The deposition is thus performed using the effective charge rather than the absolute charge,

$$
\begin{aligned}
& \rho_{p}=\frac{1}{\text { volume }} \sum_{i} \frac{q_{p i}}{1-\beta_{z i}(\xi)} \\
& \vec{J}_{p}=\frac{1}{\text { volume }} \sum_{i} \frac{q_{p i} \vec{v}_{p i}}{1-\beta_{z i}(\xi)}
\end{aligned}
$$

\section{f. Two-dimensional Wakefield Subroutine}

The velocities of-and fields resulting from-plasma particles are interrelated; calculation of one requires knowledge of the other. The method by which the simulation algorithm advances the plasma particles in time requires some additional complexity to avoid the circularity of the trajectories and fields.

The simulation of the formation of the wakefield is carried out with time steps of size $\Delta \xi$. By choice, the velocities of the plasma particles are determined at wholeinteger steps, and so the positions of the plasma particles are determined at halfinteger steps.

The objective is to determine the velocity $v_{p}$ of a given plasma particle at step $m+1$ given the velocity of the particle at step $m$. Advancing the velocity requires the fields at step $m+1 / 2$. Calculating the fields at $m+1 / 2$ requires $\rho$ and $\vec{J}$ at step $m+1 / 2$. Depositing the plasma particles at step $m+1 / 2$ requires 


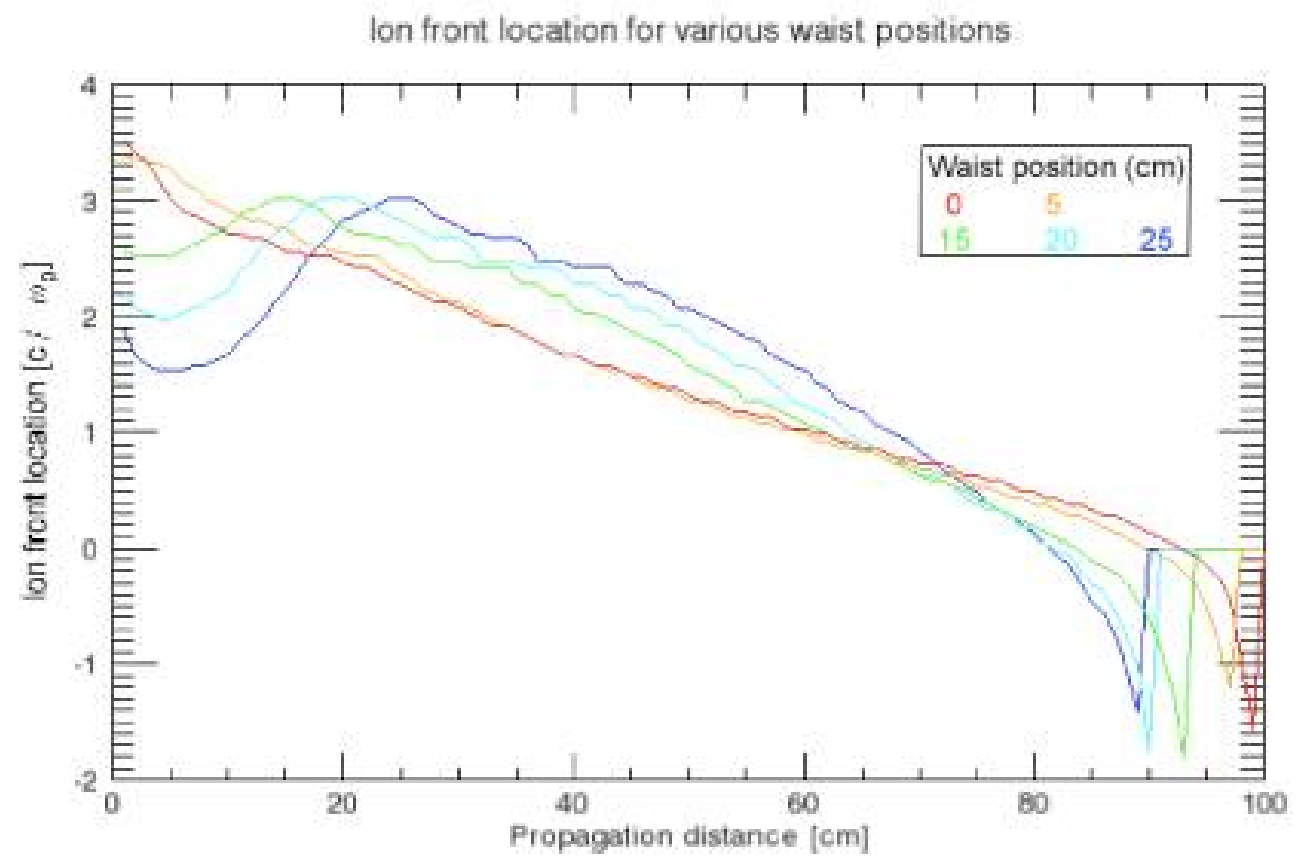

Figure 15. Ionization front, defined as minimum $\xi$ in the beam co-moving frame at which plasma charge density is non-zero. Order in which the ionization front location reduces to $\xi_{\text {ion }}<$ -1 (indicative of beam disintegration), shows that beams focused near $z=0$ propagate farther in the plasma than beams focused at larger $z$. Crossing of curves could indicate inefficient ion channel formation by deeply focused beams.

knowledge of the velocities of the plasma particles at step $m+1 / 2$. This is problematic since the velocities of the plasma particles are only determined in whole-integer steps.

To avoid this circularity in advancing the velocities of the plasma particles, the charge deposition $\rho_{p}$ and $\vec{J}_{b}$ are estimated at $m+1 / 2$ and iteratively corrected using the resulting value of $v_{p}$ at $m+1$. The strategy is to first guess $\vec{J}_{p}$ and $\rho_{p}$ at step $m+1 / 2$ so that $v_{p}$ at $m+1 / 2$ is not needed. All fields at $m+1 / 2$ are then computed using the assumed plasma charge distribution and the beam deposition, which is fixed for a given value of s. The position and momentum of each plasma particle is advanced using the evolution equations, and the velocity of $v_{p}$ of the particle at the subsequent time step $m+1$ is computed. The estimates of $\vec{J}$ and $\rho$ at the intermediate step $m+1 / 2$ are then corrected based on the new calculated value of $v_{p}$. This guess and correcting algorithm can be repeated until the desired accuracy is obtained.

\section{g. Implementation Overview}

A simplified schematic of the OuickPIC algorithm is as follows:

1. At a given $s$, deposit beam particles in $(x, y, \xi)$ space.

2. Call plasma subroutine.

(a) Initialize plasma particles at a value of $\xi$ sufficiently ahead of the beam.

(b) Push plasma particles forward in $\xi$.

i) Calculate $v_{p}$ from (32) and (33).

ii) Deposit plasma particles by calculating $\rho_{p}$ and $\vec{J}_{p}$ from (40).

iii) Calculate the fields from (22), (23), and (24)!

iv) Calculate the derivatives necessary to evolve the position and momentum of the plasma particles using (32) and (34). 


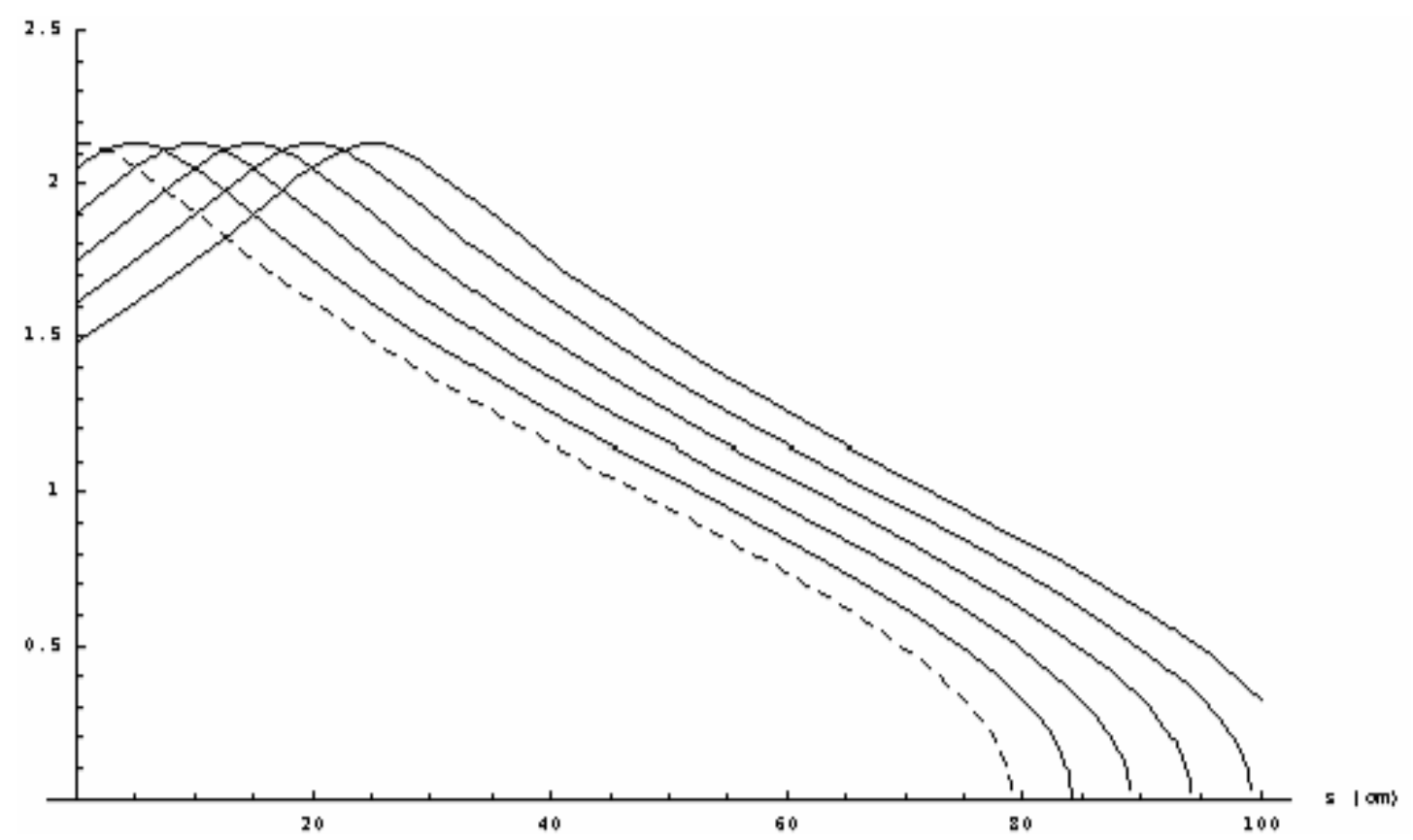

Figure 16. Theoretical prediction for ionization front evolution as a function of propagation distance [12]. Simulations assume a symmetric beam and that the drive beam focuses as if in vacuum. Theory shows general agreement with simulation (Figure 15), although theory does not predict crossing of ion front curves.

c) Repeat for each step in $\xi$.

3. After advancing the plasma particles in $\xi$, advance the beam in $s$.

4. Update the position and momentum of each beam particle and repeat.

\section{DATA}

\section{a. Simulation Parameters}

The simulations discussed in this paper use the QuickPIC algorithm to propagate an electron beam through one meter of plasma. The plasma is created by the self-ionization of a lithium vapor. The beam and plasma parameters are selected to model the conditions of the E-167 experiment. A summary of the simulation parameter values that follow is presented in Table II.

The simulated drive beam is a pulse consisting of $1.7 \times 10^{10}$ ultra-relativistic electrons. The beam is initialized to be precisely mono-energetic, with all beam electrons having relativistic boost $\gamma=$ 82192. The normalized emittances, in units of $\mathrm{mm} \cdot \mathrm{mrad}$, are 100 in the $x$ direction and 20 in the $y$ direction. The longitudinal profile of the beam is piece-wise linear and somewhat Gaussian, as shown in Figure 11. In physical units, the r.m.s. length of the drive bunch is $31.8 \mu \mathrm{m}$.

All longitudinal distances $z$ are measured relative to the plane in which the beam enters the plasma, taken to be $z=0$. The transverse beam profile is specified by the values of the Twiss parameters at $z=0$. The density of the plasma is assumed to be sufficiently small that the drive beam focuses as if propagating through a vacuum.

The plasma is created by single ionization of lithium atoms upon impact of the drive beam. The simulations presented here do not include the effect of multiple ionizations, which is expected to be small in this case due to the high secondary ionization potential of lithium. The density of the plasma is constant, $2.7 \times 10^{17} \mathrm{~cm}^{-3}$, from $z=10 \mathrm{~cm}$ onwards. Between $x=0 \mathrm{~cm}$ and $z=10 \mathrm{~cm}$, the plasma density increases linearly from 0 to $2.7 \times 10^{17} \mathrm{~cm}^{-3}$.

The only parameters that are varied between simulations are the Twiss parameters at $z=0$. By specifying the Twiss parameters, we vary the drive beam waist location from $z=0$ to $z=25 \mathrm{~cm}$. 


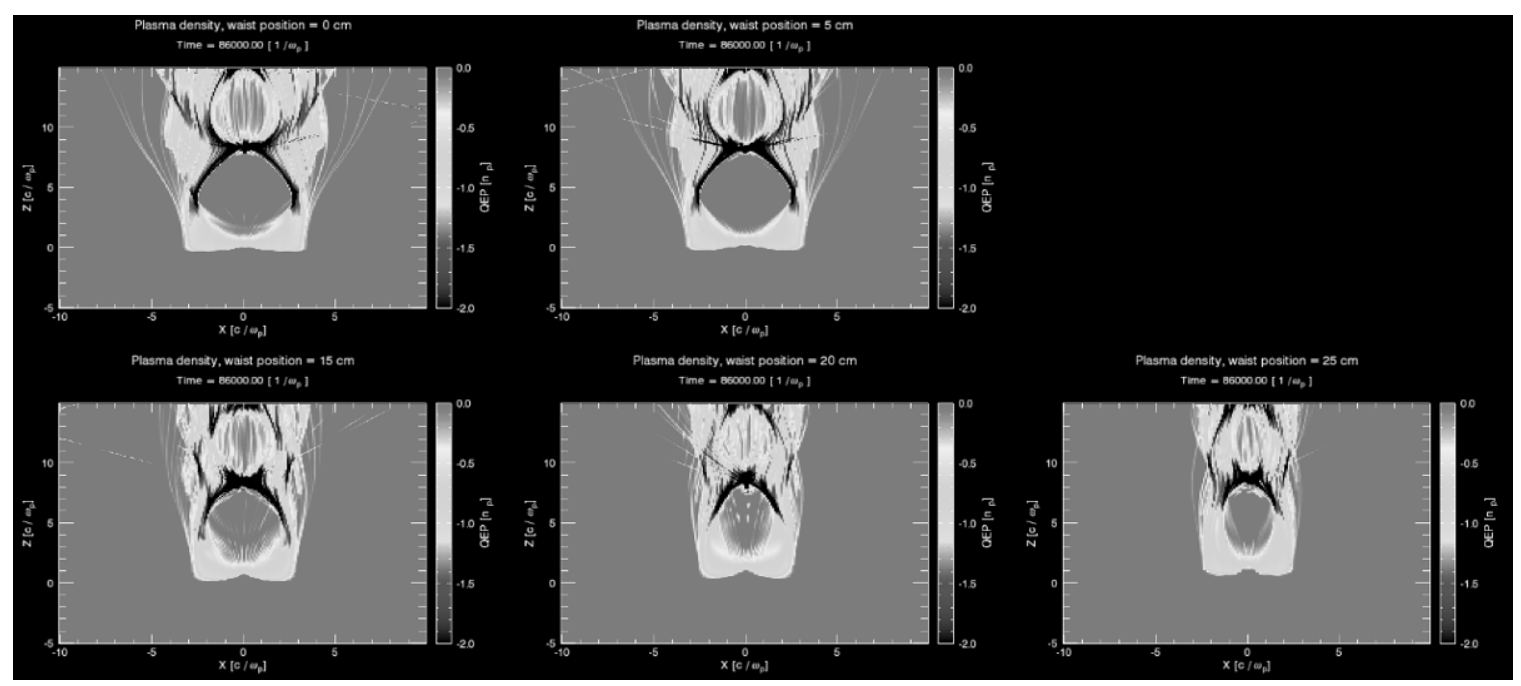

Figure 17. Representative frames of beam charge density at $z \approx 86 \mathrm{~cm}$. Each frame shows a different waist position: upper left $=0 \mathrm{~cm}$; upper right $=5 \mathrm{~cm}$; lower left $=15 \mathrm{~cm}$; lower middle $=$ $20 \mathrm{~cm}$; and lower right $=25 \mathrm{~cm}$. The blown out ion channel is smaller in the case of beams focused at large $z$. Ion channel formation may limit the energy obtained by deeply focused beams.

Unfortunately, it is not possible to simulate the case in which the waist is positioned a $z$ $=10 \mathrm{~cm}$ due to numerical instability (resulting from trapped particles).

\section{b. Diagnostics}

Unlike physical experiments, computer simulations allow exact diagnostics of beam and plasma parameters to be taken at regular intervals. The time interval of the simulation is equal to the inverse plasma frequency, $1 / \omega_{p}$. The plasma frequency is evaluated in the constant density region of the plasma, $\omega_{p} \approx 2.931 \times 10^{13} \mathrm{~s}^{-1}$. Since the beam is assumed to propagate at the speed of light, the distance traveled by the beam during each simulation time step is equal to the collisionless plasma skin depth, $c / \omega_{p} \approx 10.23$ $\mu \mathrm{m}$.

Beam charge density is sampled every two time steps, and plasma charge density is sampled every ten time steps. This choice of timescales is chosen to be consistent with the quasi-static approximation, which assumes that the time scale for evolutions of the wakefield is much less than that for the drive beam.
The ionization front is determined from the beam charge density as the plane of smallest $z$ in which the beam charge density is non-zero. The evolution of the ionization front as a function of beam propagation distance is shown in Figure 15.

Longitudinal electromagnetic fields in the plasma are measured every ten time steps in both the $x z$ and $y z$ planes. Although the length scales of the beam envelope in the two planes are different, the evolution of the fields is qualitatively very similar in the two transverse dimensions. Henceforth, we will focus of the field in the $x z$ plane.

From the electric field in $x z$ plane, we calculate the transformer ratio as a function of propagation distance, shown in Figure 19. The transformer ratio is calculated based on the longitudinal profile of $E_{z}$ in the $x=0$ plane, and is equal to the ratio of the effective accelerating field to the maximum decelerating field. The maximum value of the profile of $E_{z}$ along $x=0$ within the first bucket is taken as the maximum decelerating field. Calculating the effective accelerating field is performed in several steps. The point at which $E_{z}$ returns to 0 after achieving an initial maximum is first identified. The tangent to the profile of $E_{z}$ along $x=0$ is then estimated at this point. The effective accelerating field is taken as 


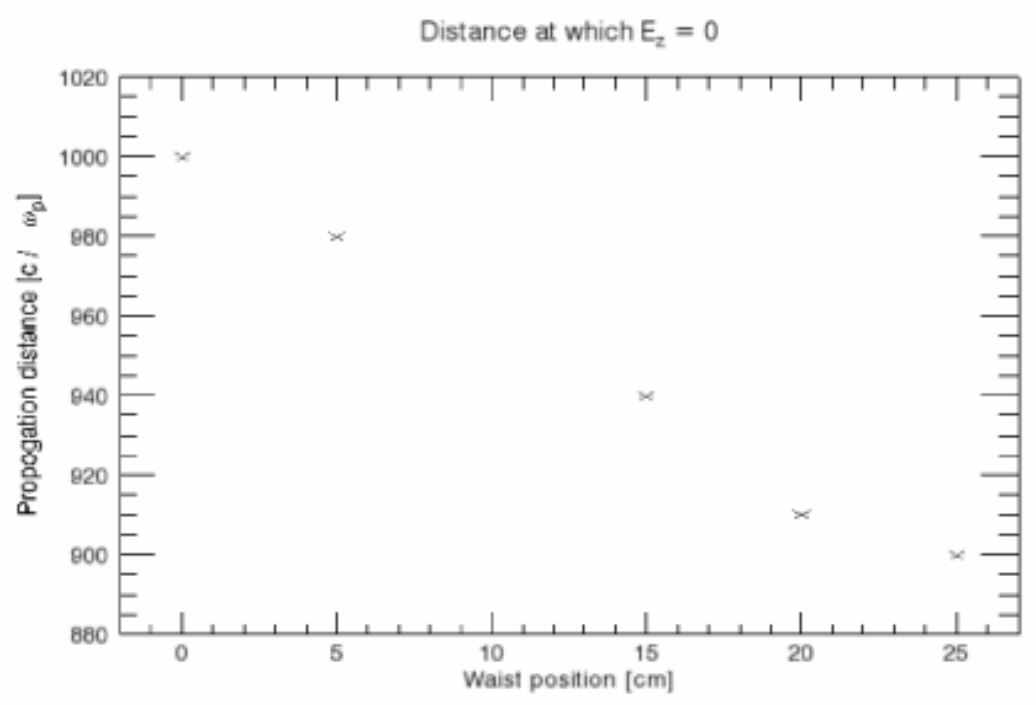

Figure 18. Location in $z$ at which $E_{z}$ in the co-moving frame of the beam becomes 0 . The electric field confirms the previous assertion that beams focused near $z=0$ propagate farther in the plasma than those focused at larger $z$.

the intersect of this tangent with the near vertical step in $E_{z}$ that occurs at the rear of the bucket.

Phase space data for the beam is output every two time steps, and contains $x$, $y, z, p_{x}, p_{y}$, and $p_{z}$. The data is recorder as continuous variables that must be binned and discretized on a grid. Figure 21 shows phase space data discretized with a mesh of 512 by 512 points. This sampling resolution is a compromise between resolution and computation time.

\section{DISCUSSION}

\section{a. Energy}

The dependence of the maximum and minimum particle energies on propagation distance is shown in Figure 12. The maximum energy achieved appears to be greatest for beams focused near $z=0$ and decreases with increasing focusing distance. The rate of energy gain of all beams is initially similar. However, energy gain produced by beam focused at larger $Z$ appears to be limited, whereas the rate of energy gain produced by the beam focused at $z=0$ is constant until disintegration of the beam. That shallowly focused beams achieve greater energy is not intuitive, since such beams tend to be more susceptible to instabilities that can detract from the energy available for acceleration.

Plotting the extrema of energy obtained over the full propagation distance of the beam in Figure 13 confirms what is suggested by figure 12: shallowly focused drive beams accelerate the witness bunch to greater maximum energy. The maximum beam energy shows a clear downward trend with increasing waist distance, whereas the minimum energy shows an increasing trend as waist distance is increased. Both of these dependences appear to be linear, although there is no a priori reason to expect this linearity.

The distance after the waist at which each extremum shown in Figure 13 is achieved is plotted in Figure 14 . The location of the extrema relative to waist location generally decreases for more deeply focused beams. This decrease is partly a simple consequence that, all other factors being equal, the distance to an extremum at constant location minus the distance to the beam waist decreases as the beam waist distance is increased. However, linear fits of the absolute maxima and minima locations, respectively, in Figure 14 indicate that there is likely a waistdependent component of the location of the extrema. The slopes of simple linear fits to the maxima and minima locations have slopes of $-1.3 \pm 0.376$ and $-1.7 \pm 0.282$, 

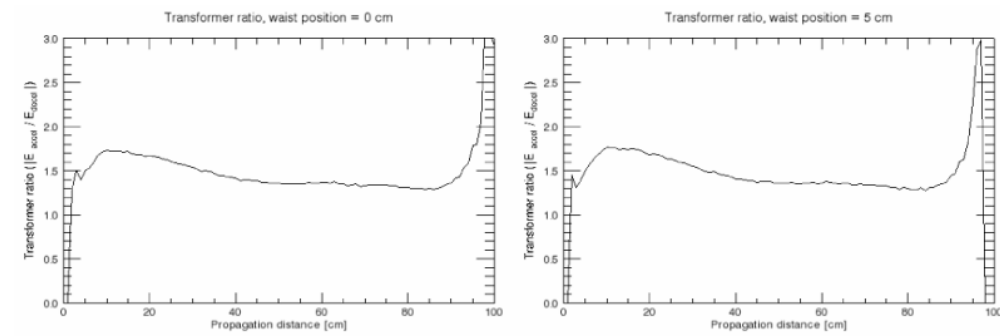

Transtorner ratio, waist postion $=15 \mathrm{~cm}$

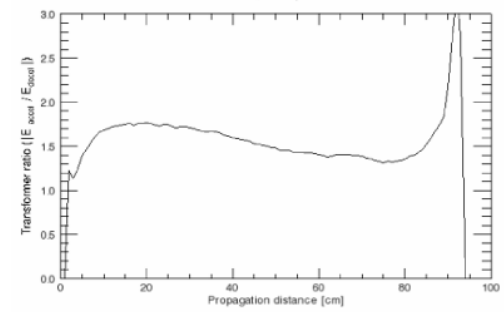

Transtormer ratio, wast postion $=20 \mathrm{~cm}$

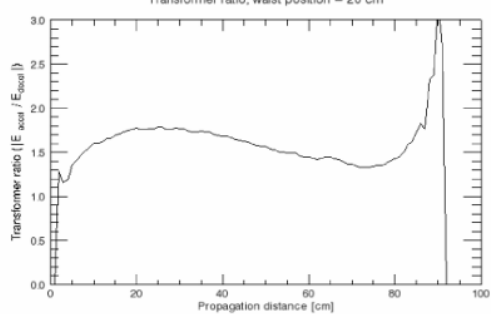

Transtormer ratio, waist postion $=25 \mathrm{~cm}$

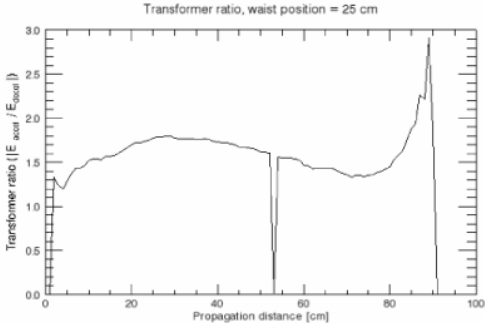

Figure 19. Transformer ratio, defined as the ratio of the effective accelerating field to the maximum decelerating field. All beams show maximum transformer ratio near the waist, consistent with a larger accelerating field due to a smaller transverse spot size. Pictures are ordered as in Figure 17, with waist position $=0 \mathrm{~cm}$ at top left. Transformer ratios of beams focused near $z=0$ are relatively constant, consistent with nearly constant change in energy maxima. The spike in the curve for a waist at $25 \mathrm{~cm}$ (lower right) is due to the computational algorithm and is not a physical effect.

respectively. If the location of extrema were independent of waist location, the relation between extrema and beam waist locations would be linear with a slope of -1 .

Figure 12 indicates that in none of the simulations presented here does the beam energy reach 0 . Hence the witness bunch energy gain does not appear to be limited by depletion of drive beam energy, a condition known as pump depletion.

The waist dependence of the energy extrema location and magnitude as well as the energy gain limitation could be due to variation in ion channel formation between beams focused at different locations. These possibilities are discussed below in relation to the ionization front.

\section{b. Ionization Front}

The ionization front is measured in the co-moving frame of the beam, in which the origin of coordinates is taken to be the center point of the initial head of the drive beam. The ionization front is defined as the smallest $\xi$ at which the plasma charge density is non-zero. The ionization front as a function of propagation distance is shown in Figure 15.

As shown in the figure, the ionization front is greatest in all cases at the location of the waist. This is consistent with (8) which predicts that the radial electric field governing ion channel formation is inversely proportional to beam transverse spot size. An ionization front at $\xi<-1$ is indicative of beam collapse. The order in which the beams in Figure 15 collapse shows that beam propagation distance decreases with increasing waist distance.

The reduction in ionization front location that occurs at small $z$ is not well understood. This reduction is not predicted by theory, and is not reproduced by Figure 16. It is possible that the location of the ionization front is affected by the linear density ramp that occurs between $z=0$ and $z=10 \mathrm{~cm}$, although it is not clear why a less dense plasma would result in decreased ionization front location. More simulations with varying density ramps are necessary to further understand this behavior.

A theoretical prediction of ionization front evolution is shown in Figure 16 [12]. This prediction is based on a symmetric 

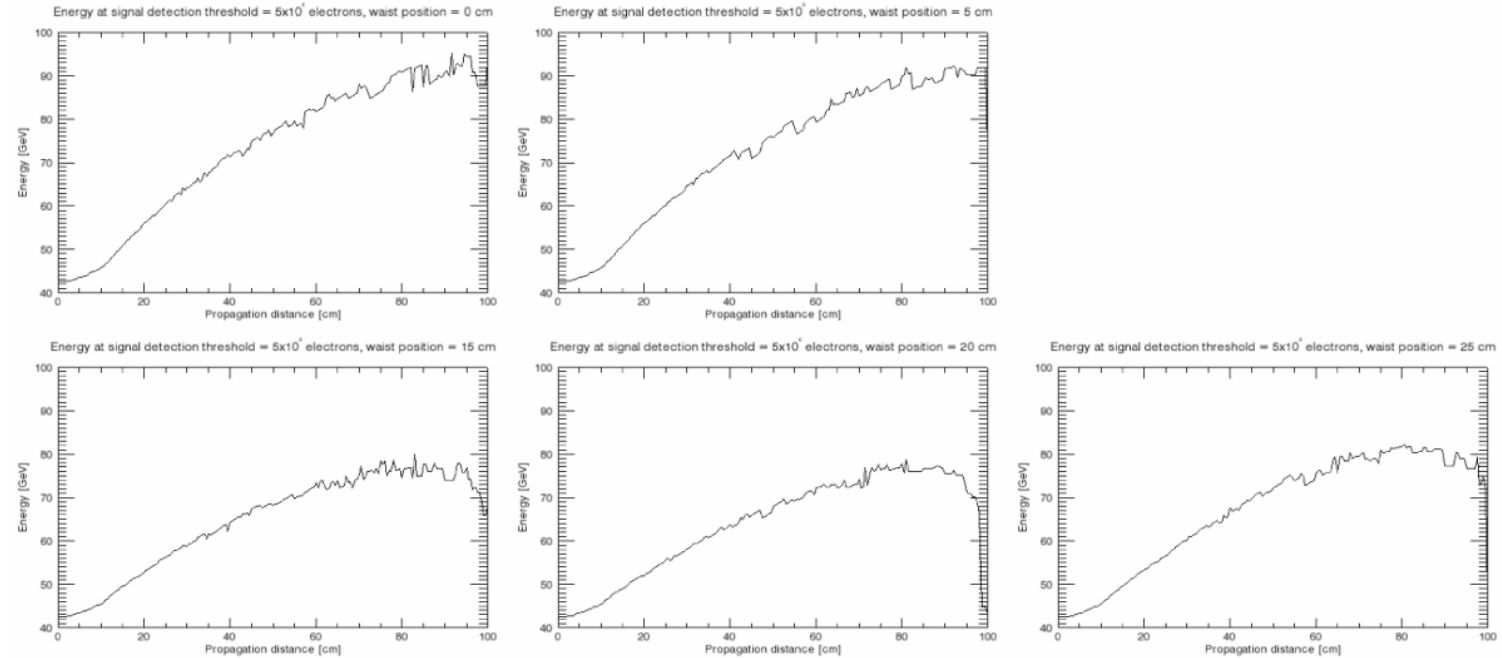

Figure 20. Maximum energy observable by the E-167 experiment apparatus. Results are similar to the simulation shown in Figure 12. Frames are ordered by waist position as in Figure 17, with waist position $=0 \mathrm{~cm}$ at top left. Maximum observable energy is smaller and change is not as constant for beams focused at small $z$; nevertheless, the energy of some particles is more than doubled.

beam with constant density, so the comparison to Figure 15 is not exact. However, there is agreement with the general pattern of ionization front evolution: increase to a maximum at the beam waist, followed by a linear decrease, culminating in a sharp decrease as the beam collapses.

However, there are several notable differences between the theoretical and observed ionization front evolution. The ionization front curves of the three simulations focused after the plasma density ramp, with waist locations at $z=15,20$, and $25 \mathrm{~cm}$ respectively, achieve approximately the same maximum magnitude at their respective waists. These curves also intersect at approximately one point. The other two simulations which are focused within the $z$ range of the plasma density ramp also have similar maximum magnitude and intersect at a single point. The maximum and intersection point of this second group is distinct from those of the group of simulations in which the beam is focused after the density ramp.

The apparent separation of the simulations into pre- and post-10 cm focusing groups may indicate the importance of the linear plasma density ramp between 0 and $10 \mathrm{~cm}$ in determining the beam behavior at all further $z$. The effect of such plasma density variations has not previously been studied in depth, but initially we did not expect the density ramp to have a significant effect on drive beam propagation.

It is notable that the ionization front curves in Figure 15 cross one another. This crossing indicates that the beam structure degrades more rapidly for beam focused at larger $z$. A beam that is focused near $z=0$ does not experience degradation comparable to that experienced by a beam focused at larger $z$ even as the spot size of the beam focused at smaller $z$ expands to be greater than that of the beam focused at larger $z$. Ion channel formation appears to be influenced by the history of the beam rather than simply the transverse spot size at a given point.

Beams that are focused at greater $z$ have larger transverse spot sizes at $z=0$. By (8) [see part one of this article in the March issue], the transverse electric field which governs ion channel formation is initially less in such beams. Because of the inefficiency with which plasma electrons are expelled from the ion channel, the head of the drive beam could experience increased erosion early in the propagation of the beam. Head erosion would further decrease the transverse electric field, resulting in a 


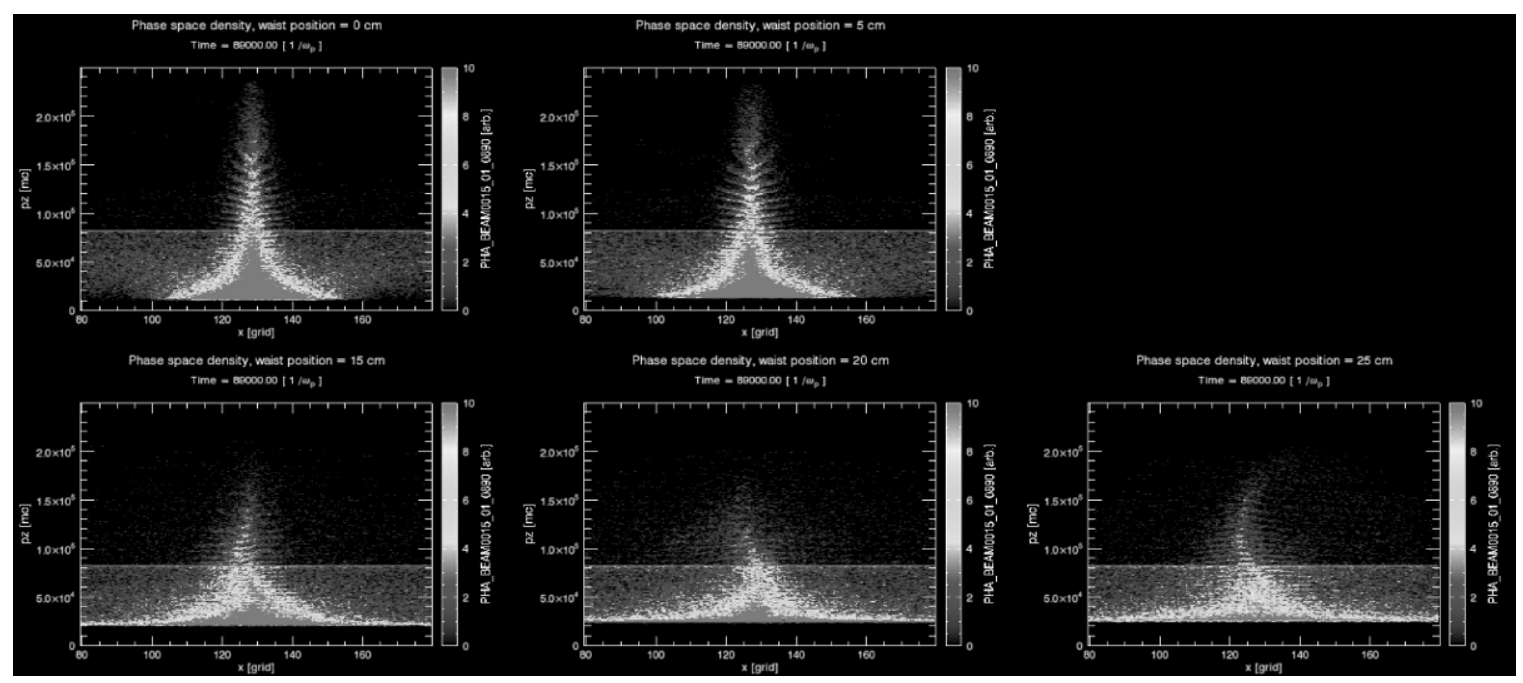

Figure 21. Phase space plots of $p_{z}$ versus $x$ from simulations. Frames are arranged as in Figure 17 , with waist position $=0 \mathrm{~cm}$ at top left. Vertical scale in these plots is proportional to the vertical scale of the experimentally observed energy spectrum (Figure 9). None of the waist locations tested reproduced the monotonic tail of very low energy particles shown present in the experimental data.

positively reinforcing cycle that could explain the reduced propagation distance of beams focused at larger $z$.

Incomplete ionization of the ion channel by a weak transverse electric field would lead to a lower acceleration gradient as well as a smaller ion channel radius. It is possible that the efficiency of the wakefield structure could be reduced by the smaller radius of ion channel or encroachment of the ionization front. Hence a self-reinforcing cycle of head erosion and incomplete ion channel formation could lead to limitations on the maximum beam energy.

Focusing of the drive beam by the plasma acceleration medium is another mechanism that could contribute to history dependence of the formation of the ion channel. Beams focused near $z=0$ would be more able to achieve a pure ion channel at small $z$ due to the smaller initial transverse spot size of such a beam. Once established, a pure ion channel could contribute to focusing of the beam due to strong radial electric fields. It would be difficult for a beam focused at larger $z$ to establish comparable transverse field that could lead to plasma focusing, since as discussed above, such beams are likely more limited by head erosion. This plasma focusing would maintain the spot size of the beam focused at small $z$ to be less than that of a beam focused at larger $z$ at $a$ comparable distance relative to the respective beam waists.

A sample of the plasma charge density is shown in Figure 17. This frame is taken from $z \approx 86 \mathrm{~cm}$, after considerable propagation distance, when differences in beam shape become more pronounced. The ionization channel of the beam focused at $z=25 \mathrm{~cm}$ is narrower and shorter than that of the beams focused at smaller $z$. More deeply focused beams also show evidence of incomplete ionization of the ion column, which would reduce the strength of the resulting accelerating field.

\section{c. Electric Field}

Observations of the electric fields within the plasma support conclusions that have already been drawn from other parameter. The location in $z$ at which the longitudinal electric field becomes identically equal to 0 for a given simulation is a measure of the maximum distance to which the drive beam propagates before disintegration. As shown in Figure 18, the beam focused at smallest $z$ propagates furthest into the plasma, and propagation distance decreases as focusing distance is increased. The relationship between beam waist location and propagation distance 

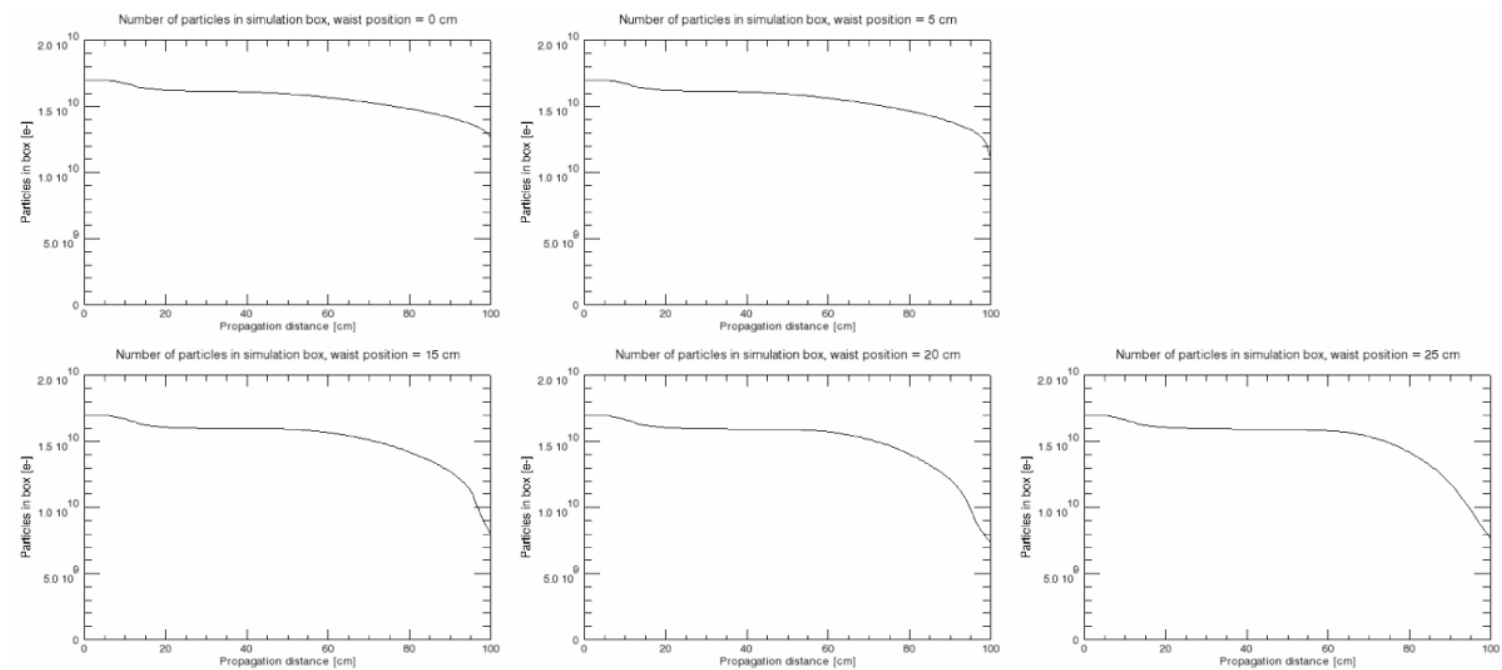

Figure 22. Particles in simulation box as a function of propagation distance. Particles lost from the simulation box could potentially be imaged by the experimental apparatus. These ejected particles would be lower energy than other beam particles and could possibly form the tail of low energy particles experimentally observed. Frames are order by waist position as in Figure 17.

appears to be linear, although there is not yet a clear theoretical motivation for this linearity.

The transformer ratio as a function of propagation distance in plotted in Figure 19. The transformer ratio for beams focused a smaller $z$ is more constant than the transformer ratio of beams focused at larger $z$. The accelerating and decelerating fields, as indicated by the line slopes of maximum and minimum energy in Figure 12, do not appear initially to be significantly greater in the case of focusing at small $z$ as opposed to large $z$. Rather, the fields are more constant over a larger range of $z$ in the case of a shallowly focused beam. Constant accelerating and decelerating fields are consistent with a more uniform transformer ratio.

\section{d. Relation to the E-167 Experiment}

Because of the limitations of experimental diagnostics, physical observations can potentially differ from simulation results. In the case of the E-167 experiment, the energy diagnostics used have minimum sensitivity, which affects the way in which the energies plotted in Figure 12 would be observed. The transverse size of the observable window is larger in the $\mathrm{E}$ 167 experiment than in the simulations presented here, and so particles that are not included in these simulation may be imaged by the E-167 experimental apparatus.

The energy diagnostics used in the E-167 experiment require about $5 \times 10^{6}$ electrons per $\mathrm{GeV}$ at a given energy in order to produce an observable signal. The maximum energy at which the simulation results achieve this energy is shown in Figure 20. Beams focused at smaller $z$ produce the largest energy gains. However, in the experimental case, the maximum observable energy is about $90 \mathrm{GeV}$ rather than $110 \mathrm{GeV}$. Observing particles with 90 $\mathrm{GeV}$ of energy would still indicate an extremely large acceleration gradient of about $50 \mathrm{GeV}$ per meter.

It appears that the beam focused at $25 \mathrm{~cm}$ may achieve greater maximum observable energy than beams with waists at 15 or $20 \mathrm{~cm}$. The beam focused at $25 \mathrm{~cm}$ may be more mono-energetic at high energy due to the fact that the limit on the maximum energy achievable by the beam focused at $25 \mathrm{~cm}$ is less than that of the beams focused at 15 and $20 \mathrm{~cm}$, respectively. Hence the beam focused at $25 \mathrm{~cm}$ appears experimentally to achieve higher energy due to a lack of particles at higher energies in unobservable small quantities.

No simulation energy spectrums, shown in Figure 21, evidence a tail of low 
energy particles like that shown in the experimental data, Figure 9. All simulations appear to have a flat-bottomed energy spectrum, with minimum energy increasing as waist distance is increased.

The plot of the beam energies provides further support for several previous assertions. That the minimum energy observed in each simulation is non-zero is indicative that acceleration does not appear to be limited by pump depletion. The beams shown in Figure 21 are beginning to degrade due to instabilities. These instabilities are terminal and lead to the eventual disintegration of the beam. The severity with which the beams are affected by instabilities increases with focusing distance, indicating that shallowly focused beams propagate farther through the plasma.

The simulations presented here suggest that the tail of the low energy particles observed experimentally is not due to the variation of drive beam waist location. These low energy particles are also not likely due to pump depletion, as the drive beam does not appear to be depleted of energy upon disintegration of the beam.

A remaining possibility for the origin of experimentally observed low energy particles is self-trapping by the wakefield structure. The simulation box used in these simulations is smaller than the experimentally observable transverse window. The number of particles in the simulation box decreases as the beam propagates in all simulations, as shown in Figure 22. It is possible that particles lost from the simulation remain within the larger experimental window. These particles that have left the simulation window will not be accelerated as effectively once ejected from the wakefield structure. Hence these particles, if imaged by the experimental energy diagnostics, could produce a tail like that shown in Figure 9. These predictions may be better confirmed as the QuickPIC simulation algorithm is improved to better model self-trapping of plasma particles.

\section{CONCLUSION}

In this paper, we present five simulations in which we vary the waist location of a beam of ultra-relativistic electrons propagating though one meter of self-ionized lithium plasma. The simulation parameters are chosen to emulate the recent Stanford Linear Accelerator Center E167 experiments, with the objective of reproducing the tail of low energy particles observed by these experiments.

We find that focusing the beam near $z=0$ results in greater maximum energy achieved by the witness bunch compared to focusing at larger $z$. Shallowly focused beams also propagate further through the plasma acceleration medium than those focused more deeply.

Maximum energy gain and propagation distance could be dependent on the effect of transverse beam spot size on ion channel formation. Beams focused at larger $z$ could experience a self-reinforcing cycle of head erosion that limits beam propagation distance and ion channel formation. Plasma focusing may maintain the transverse spot size of beams focused at smaller $z$ to be less than that of beams focused at larger $z$ at a comparable distance from the beam waist.

None of the simulations presented here show evidence of a tail of particles at very low energy, as observed in the E-167 experiments, nor do any simulations show signs of pump depletion. These negative results, and evidence of loss of simulation particles, raise the possibility that low energy particles experimentally observed could be electrons trapped within the wakefield structure.

\section{ACKNOWLEDGEMENTS}

The author would like to thank Professor W.B. Mori and Miao-miao Zhou for their guidance in preparing this research. He would also like to thanks Françoise Quéval and the National Science Foundation program Research Experiences for Undergraduates for their generosity and support.

\section{REFERENCES}

1. John M. Dawson, "Plasma Particle Accelerators," Scientific American, vol. 260 (1989) 54-61.

2. Chandrasekhar Joshi, "Plasma Accelerators," Scientific American, vol. 294, no. 2 (2006) 41-47.

3. Chandrasekhar Joshi and Thomas Katsouleas, "Particle Accelerators at the 
Energy Frontier and on Tabletops," Physics Today (June 2003) 47-53.

4. F.J. Decker, et al., "Multi-GeV Plasma Wakefield Acceleration Experiments," E167 Proposal (2005).

5. W. Lu, C. Huang, M. Zhou, W.B. Mori, and T. Katsouleas, "Nonlinear Theory for Relativistic Plasma Wakefields in the Blowout Regime," Physical Review Letters, vol. 96 (2006) 165002.

6. K. Fournier, "Recent Progress on LaserDriven X-Ray Sources" [presentation to the Department of Electrical Engineering, UCLA] 2006.

7. K.G. Steffen, High Energy Beam Optics (Interscience-Wiley, New York, 1965).

8. C. Huang, V.K. Decyk, C. Ren, M. Zhou, W. Lu, W.B. Mori, J.H. Cooley, T. M. Antonsen, and T. Katsouleas, Journal of Computational Physics (2006).

9. M. Shou, Preliminary Data from the E167 collaboration (personal communication).

10.C. Joshi, et al., Physics of Plasmas, vol. 9 (2002) 1845.
11.A.A. Geraci and D.H. Whittum, Physics of Plasmas, vol. 7 (2000) 3431.

12. Miaomiao Zhou, Development and applications of the ionization: package in QuickPIC - a novel quasi-static PIC code for PWFA study. Thesis (M.S.) UCLA, 2005.

13.P. Michel, E. Esarey, C. Schroeder, B. Shadwick, W. Leemans, "High Efficiency Electron Injection for Plasma Accelerators Using Higher-order laser Modes," Physics of Plasmas (in press).

14.D.H. Whittum, W.M. Sharp, S.S. Yu, M. Lampe, and G. Joyce, Physical Review Letters, vol. 67 (1991) 991.

15.M.J. Hogan, C.D. Barnes, C.E. Clayton, et al., Physical Review Letters, vol. 95 (2005) 54802.

16.J.B. Rosenzweig, A. Murokh, and A. Tremaine, American Institute of Physics Conference Proceedings, vol. 472 (1999) 38.

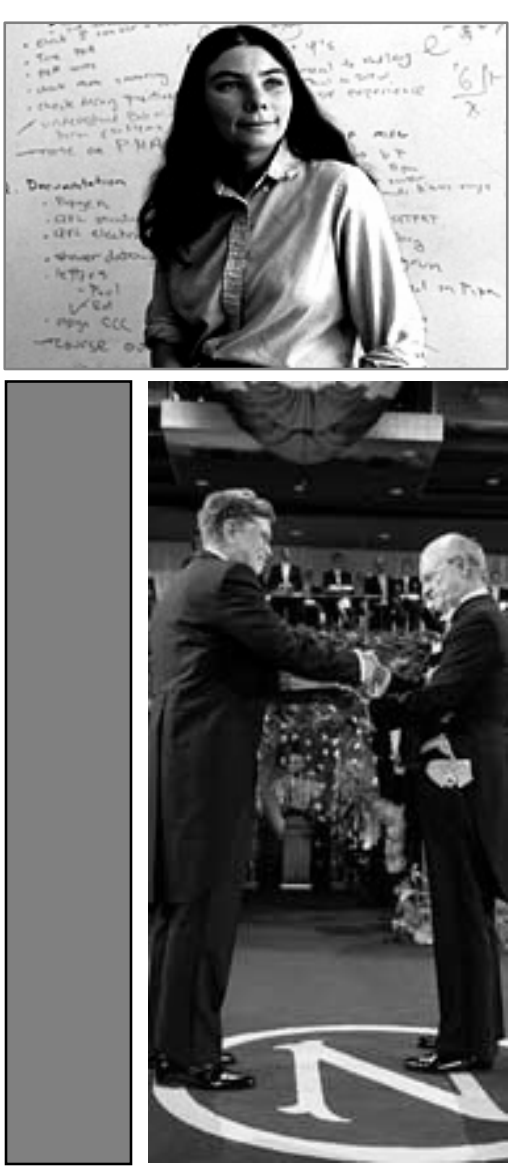

\section{UNIVERSITY OF CALIFORNIA BERKELEY}

PHYSICS AT BERKELEY has long been in the forefront of discovery and achievement. In 1931, Ernest O. Lawrence invented the cyclotron at Berkeley, ushering in the era of high-energy physics and a tradition of achievement that continues today. Seven of Berkeley's nineteen Nobel Prizes were awarded to Berkeley physicists. The most recent National Research Council nationwide rankings identify the Department as one of the best in the nation. In the last 50 years, Berkeley physicists have made many of the significant discoveries that support today's science. These discoveries extend from fundamental properties of elementary particles to spin echoes - the basis of magnetic resonance imaging - to cutting-edge breakthroughs for building an accurate model of how the universe took shape following the monster explosion commonly known as the Big Bang. Today, faculty members are leading the way to in scientific research and discovery in ways that may challenge the fundamental laws of physics particularly in the areas of gravitation, matter, and energy. At the same time, undergraduate and graduate teaching-through formal courses and research activity-is an integral part of the faculty's commitment to the development of tomorrow's scientists. In their pursuit of original research, physics faculty members collaborate with postdoctoral fellows, PhD graduate students, undergraduate students, and visiting scholars.

We believe a Physics degree represents strong training for a broad range of careers. Approximately half of our recent graduates have continued to graduate school in Physics and related fields; others have taken jobs in high tech industries or as management consultants, and still others have entered medical school or law school. We aim to help our majors develop strong mathematical and analytical skills, good laboratory skills, effective written and oral communication skills, and of course a solid understanding of the fundamental laws that govern the universe. 


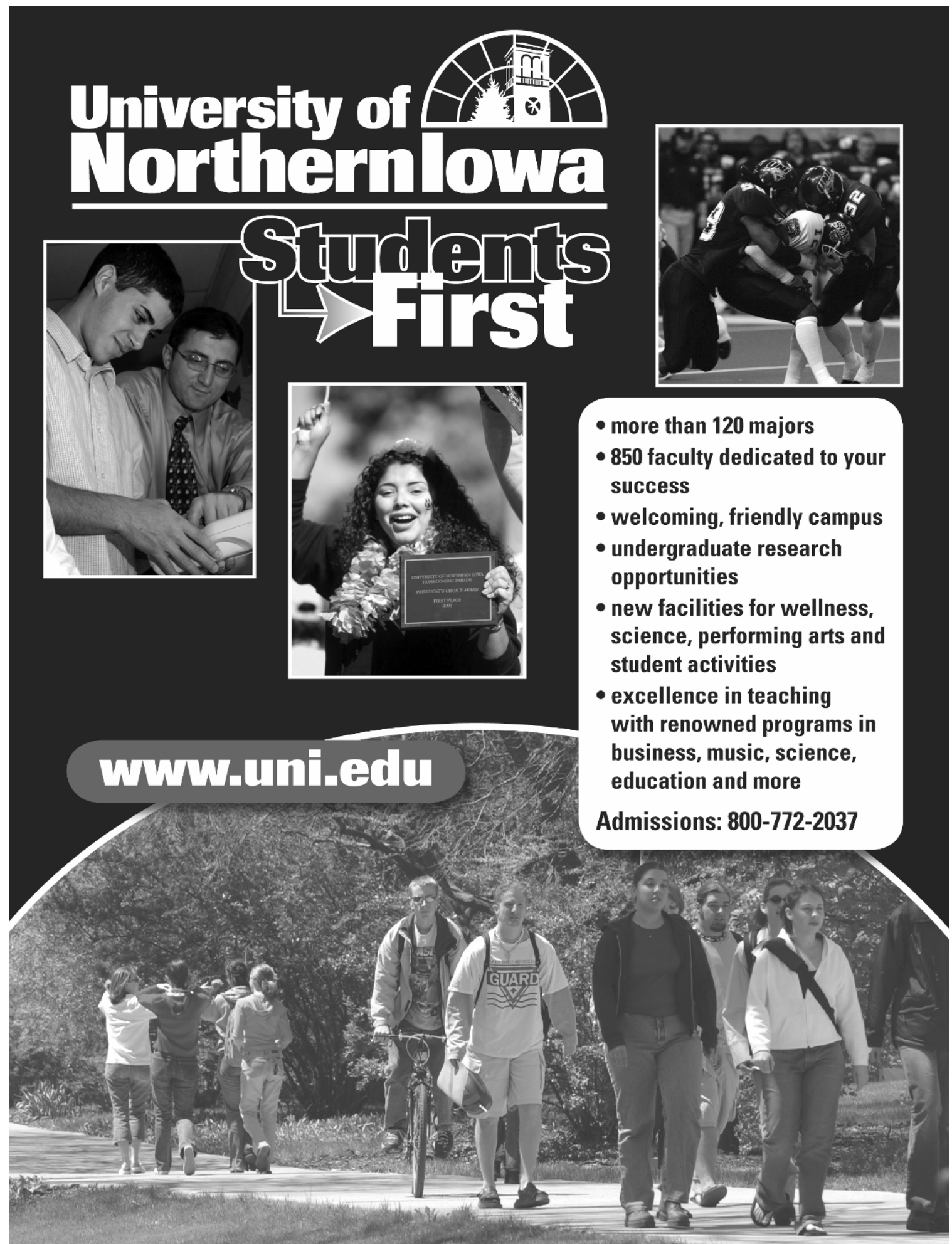

